\title{
Compound conditioning in honeybees: Blocking tests of the independence assumption
}

\author{
E. S. FUNAYAMA, P. A. COUVILLON, and M. E. BITTERMAN \\ University of Hawaii, Honolulu, Hawaii
}

\begin{abstract}
Six experiments with color-odor compounds failed to produce convincing evidence of blocking in honeybees even when the possibility of masking by within-compound association could be discounted. The parsimonious assumption that the components of a compound stimulus gain and lose associative strength independently with reinforcement and nonreinforcement of the compounds (which the experiments were designed to challenge) remains tenable for color-odor compounds, although perhaps not for intramodal compounds.
\end{abstract}

The performance of honeybees in a wide range of colorodor problems can be simulated quantitatively and with considerable accuracy on the parsimonious independence assumption - the assumption that the components of a compound stimulus gain and lose associative strength independently with reinforcement and nonreinforcement of the compound (Couvillon \& Bitterman, 1991; Fischer, Couvillon, \& Bitterman, 1993). In the vertebrate literature, the independence assumption has long been rejected in favor of the assumption that the components of a compound compete for associative strength (Rescorla \& Wagner, 1972) or for attention (Sutherland \& Mackintosh, 1971), and the question now at issue is whether the independence assumption is sufficiently constrained by the available data for honeybees.

That the functional properties of a compound stimulus may be strongly influenced by afferent interaction has been clear since the early work of Pavlov (1927) on compoundcomponent discrimination and on what Lashley (1938) later called "conditional discrimination" (discrimination between compounds made up of identical components in different combinations). Honeybees show both phenomena (Couvillon \& Bitterman, 1988). In a direct analogue of Lashley's famous experiment with rats, foraging honeybees could be trained, for example, to choose a green rather than a blue target when both targets were scented with geraniol but to choose blue rather than green when both were scented with peppermint. It should be clear, however, that evidence of interaction does not in itself contradict the independence assumption. Whether the components of a compound are altered in the process (Hull, 1943) or whether they retain their separate identities and are only supplemented by a new, compound-unique component (Rescorla, 1972; Whitlow \& Wagner, 1972), the compound still

This research was supported by Grant IBN-9308132 from the National Science Foundation. The participation of E. S. Funayama, who is now at Yale University, was made possible by an REU supplement to that grant. The authors are indebted to Estrellita T. Mateo for assistance in collection of the data. Requests for reprints should be addressed to M. E. Bitterman, Békésy Laboratory of Neurobiology, 1993 East-West Rd., Honolulu, HI 96822 (e-mail: jeffb@ahi.pbrc.hawaii.edu). can be treated as a cluster of components that are independent with respect to the influence of reinforcement and nonreinforcement.

Overshadowing is another phenomenon discovered by Pavlov (1927) that may seem to contradict the independence assumption, pointing instead to competition for associative strength or for attention. In many cases, however, simpler interpretations of the available data are at least as plausible. Consider the fact that honeybees trained with a jasmine-scented orange target respond less than do control subjects trained with an unscented orange target when both groups are tested with an unscented orange target (Couvillon \& Bitterman, 1980): the difference may reflect only the greater dissimilarity of training and testing conditions for the experimental subjects than for the controls. Or consider the fact that honeybees trained in a choice problem with color and odor confounded (e.g., green-geraniol positive versus blue-peppermint negative) learn less about the colors than do control animals trained with color relevant and odor irrelevant, and learn less about the odors than do control animals trained with odor relevant and color irrelevant (Couvillon \& Bitterman, 1989): both of these differences follow from the fact that there is little nonreinforced experience with the negative color and odor of the confounded problem, which is mastered quickly (cf. Wagner, 1969).

Competition for associative strength and for attention have been invoked also to account for blocking, a companion phenomenon to overshadowing that promises a somewhat more serious challenge to the independence assumption. The prototypical blocking experiment, designed to look for impaired conditioning of one component of a compound due to prior conditioning of another (Kamin, 1968), is adapted here in several different ways to fit the constraints imposed by work with free-flying honeybees. The general strategy is to compare null predictions based on the independence assumption with predictions of the Rescorla-Wagner theory, which is favored as a reference theory on grounds of clarity and simplicity; predictions of attention theory in the many relatively informal versions that have been proposed often are less unequivocal. 


\section{EXPERIMENT 1}

In this experiment, we looked both for blocking of odor by color and for blocking of color by odor. The subjects of an odor group were trained in the first stage of the experiment to choose between unscented green and blue targets, one color consistently reinforced and the other never. In the second stage, there was reinforced experience with targets of the two colors that were presented singly and successively, one scented with peppermint and the other with geraniol. Finally, there was a nonreinforced choice test with two gray targets, one scented with peppermint and the other with geraniol. The subjects of a color group were trained in the first stage with the scented gray targets differentially reinforced; in the second stage, they were trained as were those of the odor group; and finally they were tested with the colors. In symbolic terms, choice training with $\mathrm{A}+\mathrm{B}-$ was followed by successive training with $\mathrm{AX}+$ and $\mathrm{BY}+$, after which there was a choice test with $\mathrm{X}$ and $\mathrm{Y}$. The prediction of the Rescorla-Wagner theory is that conditioning of $X$ will be impaired by the presence of $A$ and that there will therefore be less response to $X$ than to $Y$ in the test; just such a result was, in fact, obtained by Rescorla (1981) in an autoshaping experiment with pigeons. The independence assumption provides no basis for predicting a difference in response to $\mathrm{X}$ and $\mathrm{Y}$.

\section{Method}

Subjects. The subjects were 40 foraging honeybees, all experimentally naive, from our own hives situated near the laboratory. They were assigned to odor and color groups of 12 subjects each and to two supplementary groups of 8 subjects each.

Procedure. The subjects of this and all other experiments were trained individually. In the pretraining, a forager was selected at random from a group of foragers at a feeding station providing $10 \%-12 \%$ sucrose solution and set down at a drop of $50 \%$ sucrose solution on a pretraining target that was centered on the deep sill of a laboratory window. The subject was marked with a spot of colored lacquer as it fed to repletion, after which it left for the hive. Typically, the subject would return to the laboratory after a few minutes, continuing to fly back and forth between the hive and the window as long as sucrose was available there. If the marked subject did not return after its first placement, it was taken again from the feeding station (where it usually could be found) to the pretraining target and permitted again to feed to repletion. More than two placements were rarely required. The pretraining ended after the subject had returned twice to the window of its own accord.

The targets were covered plastic petri dishes, $5.5 \mathrm{~cm}$ in diameter. Drilled in the cover of each dish, $6 \mathrm{~mm}$ from its outer circumference, was a circle of eight equally spaced holes, $5 \mathrm{~mm}$ in diameter. The dishes contained pieces of cotton batting that could be impregnated as required with the scent of peppermint or geraniol. The covers of some dishes were gray in color, others were green or blue (the standard green and blue plastics used in our modeling experiments). The covers of the dishes used in the pretraining were half green and half blue ( $180^{\circ}$ of each color) and their batting was scented with both peppermint and geraniol. Each target used on any visit was drawn from a large set of identical targets, to which it was returned after being washed at the end of the visit; the purpose of the procedure (used routinely in this laboratory) was to randomize irrelevant stimuli.

In the first stage of training, there were eight visits. Arriving from the hive on these visits, each subject in the odor group and one of the supplementary groups found a pair of unscented targets, one green and one blue, set $10 \mathrm{~cm}$ apart on the window sill. One of the two targets $(\mathrm{S}+)$ - green for half the subjects in each group and blue for the rest - contained a large $(\sim 100 \mu \mathrm{l})$ drop of $50 \%$ sucrose solution; the other target $(\mathrm{S}-$ ) contained a large drop of tap water (unacceptable to the animal and distinguishable from the sucrose solution only by taste). The lateral arrangement of the targets varied over visits in quasi-random sequence, each color half the time on the left and half the time on the right. The initial choice made by the subject on each visit was recorded, with immediate correction of error permitted, the visit ending with feeding to repletion and return to the hive. For the color group and the other supplementary group, the procedure was the same except that the training was with peppermint- and geraniolscented gray targets.

For the odor and color groups, there was a second stage of training that was the same for both. It consisted of eight reinforced visits to two targets presented singly and sequentially in balanced quasirandom orders at the center of the window sill, four visits to each target. One target was green and the other blue; one was scented with peppermint and the other with geraniol. For half of the subjects in the odor group, peppermint was the prospectively blocked odor (reinforced in compound with the $\mathrm{S}+$ color) and geraniol was the control odor (reinforced in compound with the $\mathrm{S}-$ color); for the rest, the opposite was true. (The balancing, both here and everywhere else, was factorial; each of the four possible color-odor combinations was used equally often in each group.) For half of the subjects in the color group, green was the prospectively blocked color (reinforced in compound with the $\mathrm{S}+$ odor) and blue was the control color (reinforced in compound with the $\mathrm{S}-$ odor); for the rest, the opposite was true. Each target now contained a drop of $50 \%$ sucrose solution from which there was feeding to repletion.

After its last training visit in the second stage, each subject of the odor group arrived from the hive to find a pair of gray targets, one scented with peppermint and the other with geraniol, set $10 \mathrm{~cm}$ apart in a lateral arrangement on the window sill. For half of the subjects in the group, the peppermint target was to the left of the geraniol target, and for the remaining subjects, it was to the right, each target now containing a drop of water. All actual contacts with each target during a $10-\mathrm{min}$ period were recorded by the experimenter, who pressed one of two hand-held switches that activated counters programmed to print stored frequencies at $30-\mathrm{sec}$ intervals. After the second stage of its training, each subject of the color group was tested in the same way with unscented green and blue targets.

For the subjects of the two supplementary groups, there was no second stage of training. Training in the first stage was followed by a nonreinforced 10 -min choice test with unscented green and blue targets for the supplementary group trained with the colored targets and by a like test with peppermint- and geraniol-scented gray targets for the supplementary group trained with the scented targets. The purpose of the supplementary groups was to demonstrate acquisition of the color and odor discriminations in the first stage of training by the same method used to look for evidence of blocking in the odor and color groups after their training in the second stage.

\section{Results}

In Figure 1, the performance of the supplementary groups is plotted in terms of the mean cumulative number of responses to the positive and negative colors and odors in successive $30-\mathrm{sec}$ intervals. The curves show a clear preference for $\mathrm{S}+$ of about the same magnitude in both groups, which was, of course to be expected, because the colors and odors were selected for equal salience and discriminability (Couvillon \& Bitterman, 1987, 1991). Analysis of variance (ANOVA) yielded a significant stimulus ( $\mathrm{S}+$ vs. $\mathrm{S}-$ ) effect $[F(1,14)=77.52, p<.0001]$ as well as a significant change in responding over four 2.5 -min blocks 


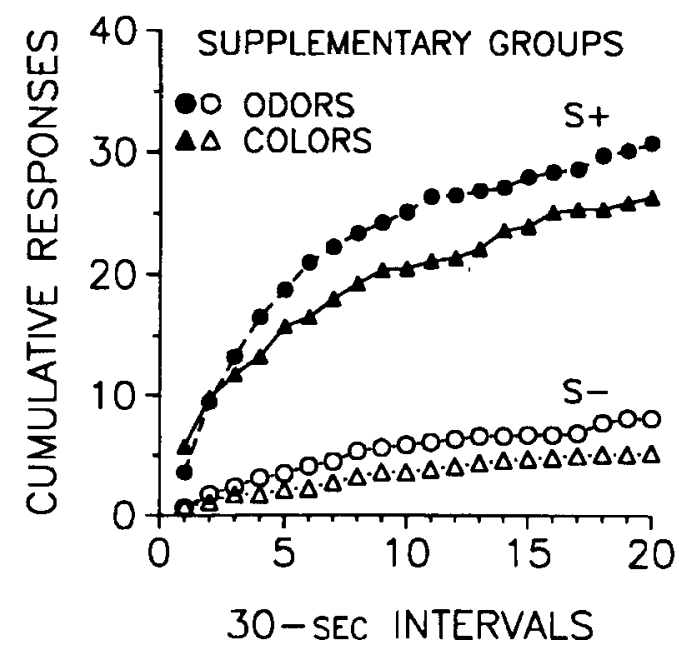

Figure 1. Performance of the supplementary groups of Experiment 1 in nonreinforced choice tests with odors and colors. One group was trained and tested with the odors; the other was trained and tested with the colors. $S+$, formerly reinforced odor or color; $\mathrm{S}-$, formerly nonreinforced odor or color.

$[F(3,42)=45.89, p<.0001]$ and a significant stimulus $\times$ block interaction $[F(3,42)=51.06, p<.0001]$, but neither a significant group $\times$ stimulus interaction $(F<1)$ nor a significant group $\times$ stimulus $\times$ block interaction $(F<1)$. It should be noted that this analysis and all subsequent analyses of like data were based on uncumulated frequencies of response (the frequencies in successive $30-\mathrm{sec}$ intervals).

In Figure 2, the performance of the color and odor groups in the choice tests is plotted in terms of the mean cumulative number of responses in successive $30-\mathrm{sec}$ intervals - the responses of the color group to each of the two colors in the lower portion and the responses of the odor group to each of the two odors in the upper portion. As the curves show, there was considerably more responding in the odor group (absent the reinforced colors) than there was in the color group (absent the reinforced odors). The difference, which is reminiscent of other such differences observed in this laboratory as well as in the early work of von Frisch (1914), can be explained most simply in terms of generalization decrement; for example, a blue-peppermint target is more similar to a gray-peppermint target than to an unscented blue target. In any case, there was not much evidence of discrimination in either group, with the larger of the differences (for the color group) opposite in direction to that which might have indicated blocking. ANOVA yielded a significant group effect $[F(1,22)=12.29, p=.0020]$ and a significant change in responding over 2.5 -min blocks $[F(3,66)=$ $34.37, p<.0001$ ] but not a significant stimulus (reinforced in compound with $\mathrm{S}+$ vs. $\mathrm{S}-)$ effect $(F<1)$, significant stimulus $\times$ block interaction $(F<1)$, significant group $\times$ stimulus interaction $[F(1,22)=2.63, p>.05]$, or significant group $\times$ stimulus $\times$ block interaction $(F<1)$. The results provide no basis for rejecting the independence assumption.

\section{EXPERIMENT 2}

In this and the subsequent experiments to be reported here, we looked only for the blocking of odor by color; where parallel experiments were carried out (as they were in several cases), the level of responding to color in the tests continued to be relatively low and open, therefore, to the suggestion that the tests might not be sufficiently sensitive. The prospective blocking experience in the first stage of the present experiment involved unscented green and blue targets that were presented singly and successively with two different magnitudes of reinforcement $(50 \%$ and $20 \%$ sucrose solutions) selected to generate two different levels of associative strength (Couvillon \& Bitterman, 1984; Loo \& Bitterman, 1992). In the second stage, the successive training of a single experimental group continued as before, except that one color was compounded with the odor of peppermint and the other with the odor of geraniol; the sucrose concentration in both cases was $50 \%$. Finally, there was a choice test with two gray targets, one scented with peppermint and the other with geraniol. In symbolic terms, training with A-50\% and B- $20 \%$ was followed by training with $\mathrm{AX}-50 \%$ and $\mathrm{BY}-50 \%$, after which there was a choice test with $\mathrm{X}$ and $\mathrm{Y}$. The prediction of the Rescorla-Wagner theory is that $\mathrm{Y}$, the odor reinforced in compound with the $20 \%$ color, will be preferred to $\mathrm{X}$, the odor reinforced in compound with the $50 \%$ color, while the independence assumption provides no basis for predicting a difference in response to the two odors.

\section{Method}

Subjects. The subjects were 20 foraging honeybees, all experimentally naive, from our own hives situated near the laboratory. They were assigned to an experimental group of 12 subjects and a supplementary group of 8 subjects.

Procedure. The subjects were recruited and pretrained in the same manner as in the previous experiment. In the first stage of training for all the animals, there were eight visits to an unscented green target

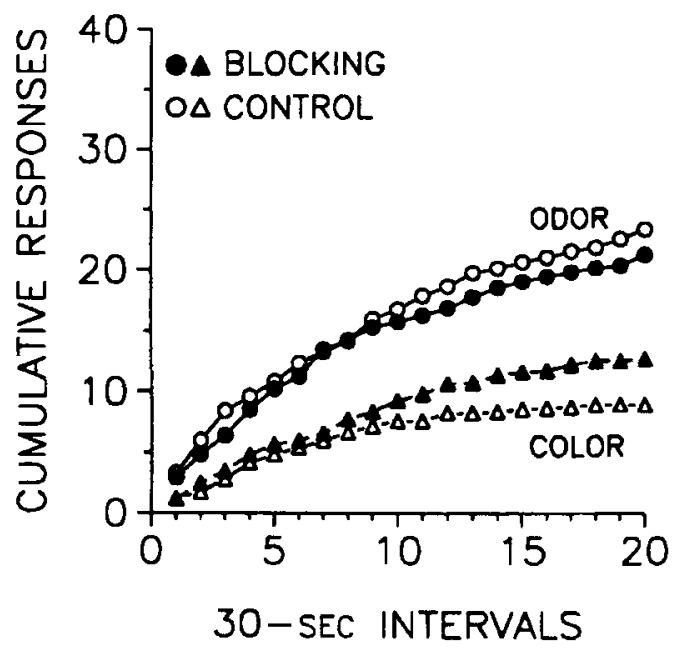

Figure 2. Performance of the odor and color groups of Experiment 1 in nonreinforced choice tests with the prospectively blocked and control alternatives. 
and eight visits to an unscented blue target in balanced quasi-random orders. The target presented on any given visit contained a drop of either $50 \%$ or $20 \%$ sucrose, from which there was feeding to repletion; green was the $50 \%$ color for half of the subjects and blue the $50 \%$ color for the rest. At the conclusion of training in the first stage, the subjects of the supplementary group had a nonreinforced 10-min choice test of the standard kind with unscented targets of the two colors (containing drops of water) that was intended simply to demonstrate anew the effectiveness of the discriminative training procedure. For the experimental group, there was a second stage of training that was like the first except that odors were added to the colors. For half of the subjects, peppermint was compounded with the previous $50 \%$ color and geraniol with the previous $20 \%$ color; the opposite was true for the rest, although the targets of both kinds now contained a $50 \%$ sucrose solution. After the second stage of training, there was a nonreinforced 10-min choice test with peppermint- and geraniolscented gray targets containing drops of water.

\section{Results}

The differential acceptability of the two sucrose concentrations was evidenced, as in previous work (Couvillon $\&$ Bitterman, 1984), by feeding patterns. Upon encountering a $20 \%$ drop of sucrose, especially after a $50 \%$ visit (that is, when it was adapted to the higher concentration), the animal would break off contact with it repeatedly before settling down to continuous feeding. Further evidence of discrimination is provided by the performance of the supplementary group when tested with the colors. A clear preference for the $50 \%$ color is shown in Figure 3, which is plotted in terms of the mean cumulative number of responses to each color in successive 30-sec intervals. ANOVA yielded a significant stimulus $(50 \%$ color vs. $20 \%$ color) effect $[F(1,7)=22.38, p=.0021]$ as well as a significant change in responding over four 2.5 -min blocks $[F(3,21)=15.24, p<.0001]$ and a significant stimulus $X$ block interaction $[F(2,21)=8.44, p=.0007]$.

In Figure 4, the performance of the experimental group in the choice test is plotted in terms of the mean cumulative number of responses to each odor-the prospectively blocked odor and the control odor--in successive 30-sec intervals. ANOVA yielded a significant change in respond-

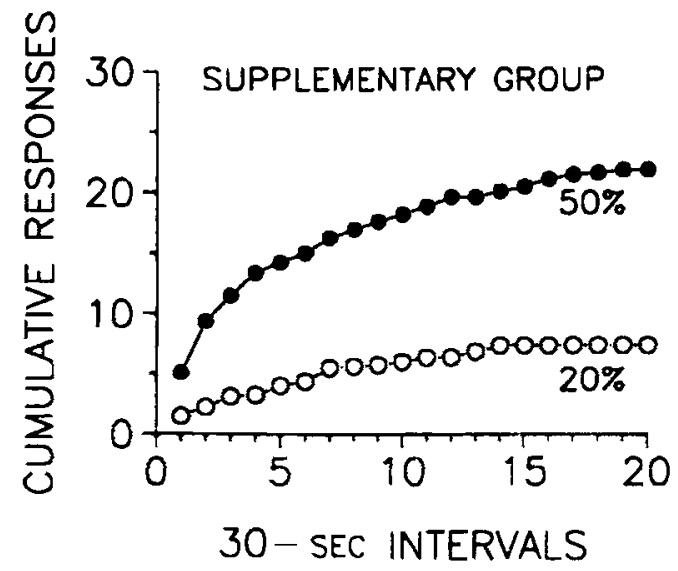

Figure 3. Performance of the supplementary group of Experiment 2 in a nonreinforced choice test with colors previously paired with $50 \%$ versus $20 \%$ sucrose solution.

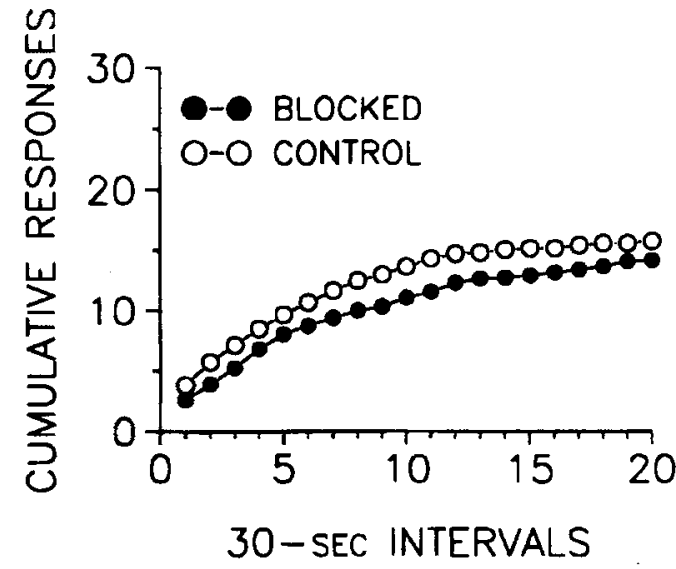

Figure 4. Performance of the experimental group of Experiment 2 in a nonreinforced choice test with the prospectively blocked and control odors.

ing over four 2.5-min blocks $[F(3,33)=36.66, p<.0001]$. The small overall difference in response to the odor reinforced in compound with the $50 \%$ color as compared with the odor reinforced in compound with the $20 \%$ color was not significant $[F(1,11)=1.39, p>.05]$, but there was a significant stimulus $\times$ block interaction $[F(3.33)=3.64$, $p=.0225]$ that might perhaps be taken as evidence of blocking.

\section{EXPERIMENT 3}

If the meager indication of blocking found in Experiment 2 was more than a statistical aberration, it should be possible, with some reasonable changes in procedural parameters, to find a more substantial effect. In Experiment 3, we trained and tested a group of subjects in the same way as the experimental group of Experiment 2, except that the total number of training visits was reduced by half. With fewer visits, we expected that there might be somewhat more responding in the test, leaving more room in which to see a blocking effect. The expectation was based on the demonstration, in honeybees, of a phenomenon that looks like the vertebrate overlearning-extinction effect the tendency for resistance to extinction first to increase but then to decrease as the number of training trials increases - and that seems to have both a specific and a generalized component (Couvillon \& Bitterman, 1984; Shinoda \& Bitterman, 1987). The data suggested that as few as four visits to each color would produce both a sharp discrimination between them and a substantial number of responses in the nonreinforced test. The logic of the third experiment was otherwise the same as that of the second.

\section{Method}

Subjects. The subjects were 12 foraging honeybecs, all experimentally naive, from our own hives situated near the laboratory.

Procedure. The subjects were recruited, traincd, and tested in the same way as those of the experimental group of Experiment 2. except that there were only four (rather than eight) visits to each coior 
in the first stage and only four (rather than eight) visits to each colorodor compound in the second stage.

\section{Results}

In Figure 5, performance in the choice test with the prospectively blocked and control odors is plotted in terms of the mean cumulative number of responses to each odor in successive $30-\mathrm{sec}$ intervals. ANOVA yielded a significant change in responding over four 2.5 -min blocks $[F(3,33)=$ $44.43, p<.0001]$, but neither a significant stimulus effect $(F<1)$, nor a significant stimulus $\times$ block interaction $[F(3,33)=1.41, p>.05]$ that is, no indication of a blocking effect. It should be noted, however, that the level of responding in the test was no greater than in the previous experiment, the total number of training visits ( 16 in all) being still great enough to generate a substantial overlearningextinction effect.

\section{EXPERIMENT 4}

The critical comparisons in the previous two concentration experiments were within-group comparisons, but here the comparison was between groups, which it was hoped might put the effects of the two treatments in clearer perspective. The between-groups design meant also that the number of training visits in the second stage was further reduced. There still were four visits to each color in the first stage of training, but in the second stage only four visits to one compound for each group rather than four to each of two compounds, bringing the total number of visits still closer to the point at which responding has been found to be maximized in overlearning-extinction experiments (Couvillon \& Bitterman, 1984). In symbolic terms, training with $A-50 \%$ and $\mathrm{B}-20 \%$ was followed by training with $\mathrm{AX}-50 \%$ for a blocking group and with $\mathrm{BX}-50 \%$ for a control group, after which both groups were tested with $\mathrm{X}$. The prediction of the Rescorla-Wagner theory is that the first group will respond less to $X$ in the test than will the second group, while the independence assumption provides no basis for predicting a difference between the groups.

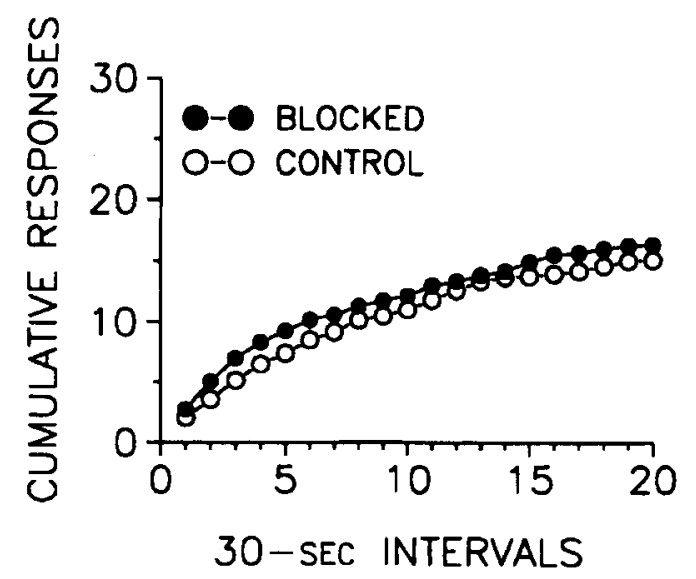

Figure 5. Performance in a nonreinforced choice test with the prospectively blocked and control odors of Experiment 3.

\section{Method}

Subjects. The subjects were 24 foraging honeybees, all experimentally naive, from our own hives situated near the laboratory. They were assigned to two groups of 12 subjects each, the blocking group and the control group.

Procedure. The subjects were recruited and pretrained in the same way as in previous concentration experiments, and with the same color-concentration pairings in the first stage of training. As in Experiment 3, there were four visits to an unscented green or blue target containing 50\% sucrose and four visits to an unscented target of the alternative color containing $20 \%$ sucrose. In the second stage, there were four visits to a peppermint-scented target containing $50 \%$ sucrose that was labeled with the $50 \%$ color of the first stage for the blocking group and with the $20 \%$ color of the first stage for the control group. Then, for the subjects of each group, there was a 10-min test with a single peppermint-scented gray target containing a drop of water.

\section{Results}

In Figure 6, the performance of the two groups in the extinction test is plotted in terms of the mean cumulative number of responses in successive 30 -sec intervals. The curves give no indication whatever of blocking. ANOVA yields a significant change in responding over four $2.5-\mathrm{min}$ blocks $[F(3,66)=38.71, p<.0001]$ but neither a significant group effect $(F<1)$ nor a significant group $\times$ block interaction $(F<1)$.

\section{EXPERIMENT 5}

In this experiment, a concurrent blocking design was employed along with a control procedure that would be expected on the basis of the Rescorla-Wagner theory to markedly enhance conditioning of the target stimulus (Rescorla, 1971; Wagner, 1971). The subjects of a blocking group had reinforced visits both to a color-odor compound and to the same color unscented, as well as nonreinforced visits to a second unscented color. For the subjects of a control group, the procedure was the same, except that the color of the compound was not reinforced when it was unscented and the unscented second color was reinforced instead. Then both groups were tested with the odor. In symbolic terms, the blocking group was trained with $\mathrm{AX}+$, $\mathrm{A}+$, and $\mathrm{B}-$, while the control group was trained with $\mathrm{AX}+, \mathrm{A}-$, and $\mathrm{B}+$, after which both groups were tested with $X$. (The role of $B$ in this design is, of course, to equate the number of reinforcements and the number of nonreinforcements for the two groups.) The prediction of the Rescorla-Wagner theory is that A+ visits will reduce the associative strength of $X$, which should approach zero, while $\mathrm{A}-$ visits will increase the associative strength of $\mathrm{X}$, which should approach its asymptotic value, with the result that the blocking group will respond less to $\mathrm{X}$ in the test. The independence assumption gives no reason to predict that the two groups will respond differently to $\mathrm{X}$.

\section{Method}

Subjects. The subjects were 32 foraging honeybees, all experimentally naive, from our own hives situated near the laboratory. They were assigned to blocking, control, and two supplementary groups of 8 subjects each. 


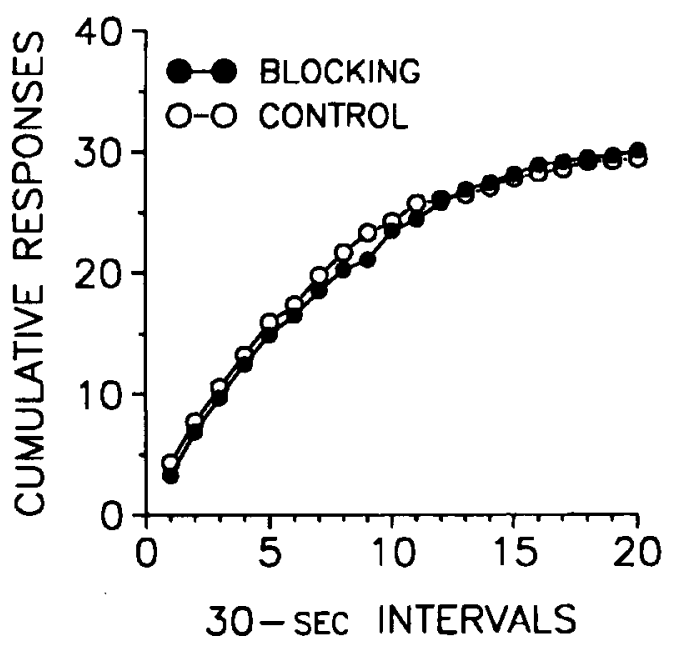

Figure 6. Performance of the blocking and control groups of Experiment 4 in nonreinforced tests with the odors.

Procedure. The subjects were recruited and pretrained in the same manner as in the previous experiments. The training was done in 16 visits, 8 of which (in balanced quasi-random orders) were to a single colored target (green for half of the animals in each group and blue for the rest) that was scented with peppermint. The target was centered on the window sill and contained a drop of $50 \%$ sucrose solution from which there was feeding to repletion. On the remaining 8 visits, the subjects were trained to discriminate between unscented targets of the two colors, with the color of the compound positive for the blocking group and one of the supplementary groups (the supplementary blocking group) and the alternative color positive for the control group and the other supplementary group (the supplementary control group). To ensure substantial experience with the negative color, a powerful discrimination procedure developed in earlier work with honeybees (Couvillon \& Bitterman, 1980) was employed. Each of the 8 discrimination-training visits began with presentation of a target of the negative color containing a drop of water. After a set delay ( $1 \mathrm{~min}$ on the first visit, $1.5 \mathrm{~min}$ on the second and $2 \mathrm{~min}$ on the rest), the first target was replaced with a target of the positive color containing a drop of $50 \%$ sucrose solution, from which there was feeding to repletion.

When each animal of the supplementary groups arrived from the hive after the last training visit, it was given a nonreinforced $10-\mathrm{min}$ choice test with unscented green and blue targets containing drops of water. The purpose of the test was to demonstrate the efficacy of the discriminative training procedure. Each animal of the blocking and control groups had a nonreinforced 10 -min period of exposure to a peppermint-scented gray target containing a drop of water.

\section{Results}

Evidence of the effectiveness of the color-discrimination training is provided by Figure 7, in which the performance of the two supplementary groups in the choice test is plotted in terms of the mean cumulative number of responses to their positive and negative colors in successive $30-\mathrm{sec}$ intervals. ANOVA yields a significant stimulus (S+ vs. $\mathrm{S}-$ ) effect $[F(1,14)=61.41, p<.0001]$, a significant change over 2.5 -min blocks $[F(3,42)=26.64, p<$ $.0001]$, and a significant stimulus $\times$ block interaction $[F(3,42)=23.18, p<.0001]$, but not a significant group effect $(F<1)$, significant group $\times$ stimulus interaction
$(F<1)$, or significant group $\times$ stimulus $\times$ block interaction $(F<1)$.

In Figure 8, the performance of the blocking and control groups in the odor test is plotted in terms of the mean cumulative number of responses in successive 30 -sec intervals. The curves show substantial levels of responding with no indication of a difference due to the treatments. ANOVA yields a significant change in responding over 2.5min blocks $[F(3,42=22.67, p<.0001]$ but not a significant group effect $(F<1)$ or a significant group $\times$ block interaction $(F<1)$. The results give no reason to question the independence assumption.

\section{EXPERIMENT 6}

The possibility now to be considered is that blocking may be masked in such experiments by within-compound association, which is found in honeybees (Couvillon \& Bitterman, 1982) as well as in vertebrates (Rescorla \& Cunningham, 1978). In Experiment 1, for example, training with $\mathrm{AX}+$ and $\mathrm{BY}+$ after $\mathrm{A}+\mathrm{B}-$ training may have enhanced the attractiveness of $X$ relative to $Y$ because opportunity was provided for within-compound association of $\mathrm{X}$ with the more excitatory $\mathrm{A}$ and of $\mathrm{Y}$ with the less excitatory B. Profiting from examples provided by vertebrate work on overshadowing and unblocking (Durlach \& Rescorla, 1980; Rescorla \& Colwill, 1983), we set out in this experiment to minimize the contribution of withincompound association by the use of an interpolated extinction procedure. The design was the same as in the first two stages of Experiment $1(\mathrm{~A}+\mathrm{B}-$ training in the first stage and training with $\mathrm{AX}+$ and $\mathrm{BY}+$ in the second stage), but the subsequent choice test with $X$ and $Y$ was preceded by nonreinforced trials with $\mathrm{A}$ and $\mathrm{B}$ that were designed to minimize their differential attractiveness.

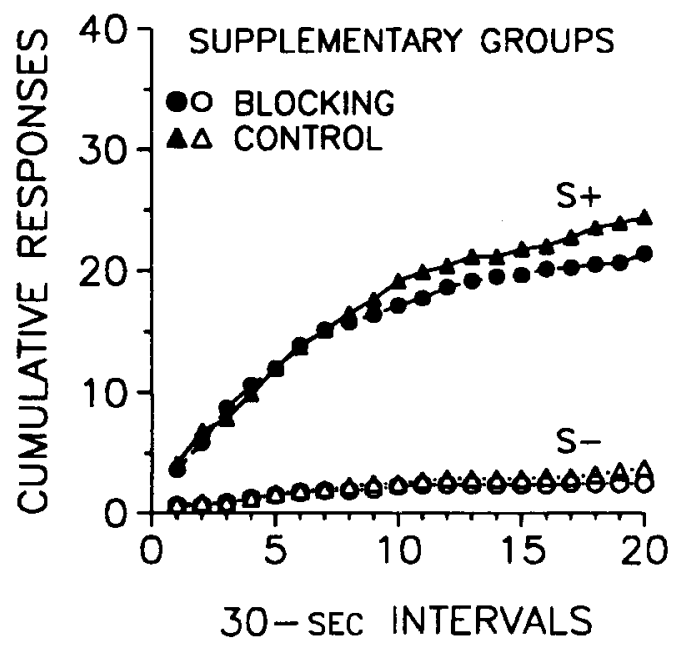

Figure 7. Performance of the supplementary groups of Experiment 5 (one trained as was the blocking group and the other as was the control group) in a nonreinforced choice test with the colors. $\mathrm{S}+$, formerly reinforced color; $\mathbf{S}-$, formerly nonreinforced color. 


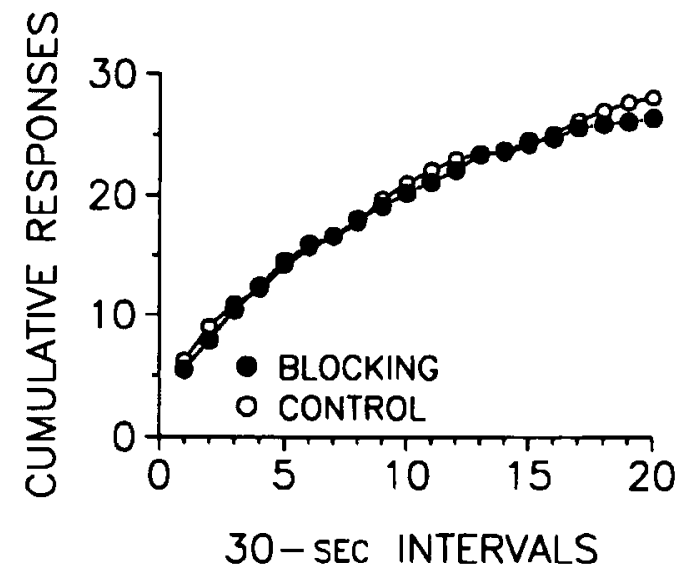

Figure 8. Performance of the blocking and control groups of Experiment 5 in a nonreinforced test with odor.

\begin{abstract}
Method
Subjects. The subjects were 8 foraging honeybees, all experimentally naive, from our own hives situated near the laboratory.

Procedure. The training in the first two stages of this experiment was the same as for the odor group of Experiment 1, after which there was a third stage of training that consisted of eight visits. Each visit began with a nonreinforced choice test with unscented blue and green targets containing drops of water, the lateral arrangement of the two colors balanced over subjects and visits. After I min, the two colored targets were replaced with a single unscented gray target containing a drop of $50 \%$ sucrose solution from which there was feeding to repletion. This procedure was designed to extinguish the preference for the positive color that had been established in the first stage of the training without at the same time discouraging return to the experimental situation. The measure of performance was the number of responses to each of the colors $\left(\mathrm{S}^{+}\right.$and $\mathrm{S}-$ ) on each visit, registered as usual by counters programmed to print stored frequencies at $30-\mathrm{sec}$ intervals. In the final stage of the experiment, there was a nonreinforced 10 -min choice test with the odors exactly as for the odor group of the first experiment.
\end{abstract}

\section{Results}

Figure 9 is plotted in terms of the mean number of responses to the colors in successive 30 -sec intervals (two intervals for each of eight visits) in the third stage of the experiment, when both colors were nonreinforced. The substantial initial preference for the formerly positive color shows that the effects of differential reinforcement in the first stage did, in fact, persist throughout the second stage, and the decline in the preference with continued extinction training in the third stage shows the effectiveness of that training. ANOVA yielded a significant stimulus ( $\mathrm{S}^{+}$ vs. $\mathrm{S}-$ ) effect $[F(1,7)=33.29, p=.0007]$ as well as a significant change in responding over four 2-min blocks $[F(3,21)=8.61, p=.0006]$ and a significant stimulus $\times$ block interaction $[F(3,21)=7.26, p=.0016]$.

In Figure 10, performance in the terminal odor test is plotted as usual in terms of the mean cumulative number of responses in successive 30 -sec intervals. ANOVA yielded a significant change in responding over four 2.5-min blocks $[F(3,21)=16.60, p<.0001]$ but neither a significant stimulus effect $(F<1)$ nor a significant group $\times$ stimulus interaction $(F<1)$. Even with the possibility of a confounding effect of within-compound association minimized, the results give no evidence of blocking.

If there actually is no blocking, it is necessary to ask why a preference for the prospectively blocked alternative was not found in Experiment 1. The design of Experiment 1 was like the design of experiments that have shown withincompound association in honeybees (Couvillon \& Bitterman, 1982), except that the training with $\mathrm{AX}+$ and $\mathrm{BY}+$ followed the training with $\mathrm{A}+\mathrm{B}-$ in Experiment 1 rather than preceding it as in the earlier experiments. That the order of training experiences should be important is not difficult to appreciate. For example, the representation of B hypothetically evoked by $\mathrm{Y}$ in the test might well be expected to be less excitatory after training with $\mathrm{BY}+$ followed by $\mathrm{B}-$ training than it would be in the reverse order. The excitatory value of $Y$ itself might also be expected to be less, because representations of $Y$ are nonreinforced in $B$ - training that follows BY+ training. Amount of training seems also to be an important variable. In one of their experiments on within-compound association, Couvillon and Bitterman (1982) found no difference in response to $X$ and $Y$ with four presentations of each compound, but a clear preference for $\mathrm{X}$ when the number of training trials was doubled. It is clear, in any case, that there still is much to be learned about within-compound association in honeybees.

\section{DISCUSSION}

Our interest in these experiments lay in the possibility of demonstrating that prior experience with one component of a color-odor compound might influence the conditioning of the other when the compound was subsequently reinforced. The various experimental treatments were expected, on the basis of the Rescorla-Wagner principle of shared associative strength, to impair (block) the conditioning of the target component, and the control treatment was in one case expected, on the basis of the same principle, even to enhance it. Only one of the six experiments

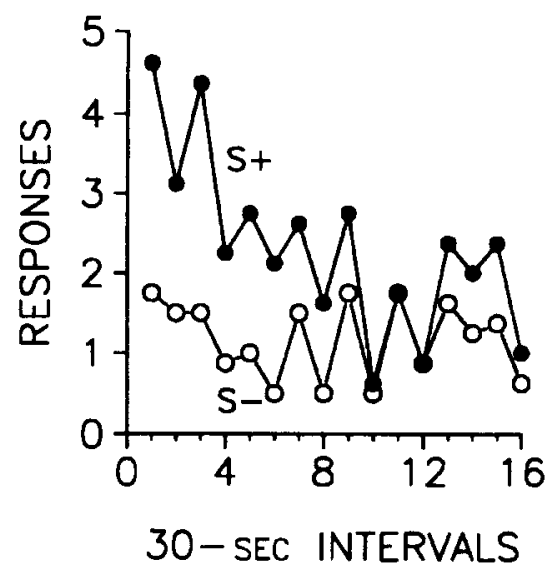

Figure 9. Performance in the third stage of Experiment 6 on nonreinforced choice trials with the two colors that had been differentially reinforced in the first stage. $S+$, formerly reinforced color; $S-$, formerly nonreinforced color. 


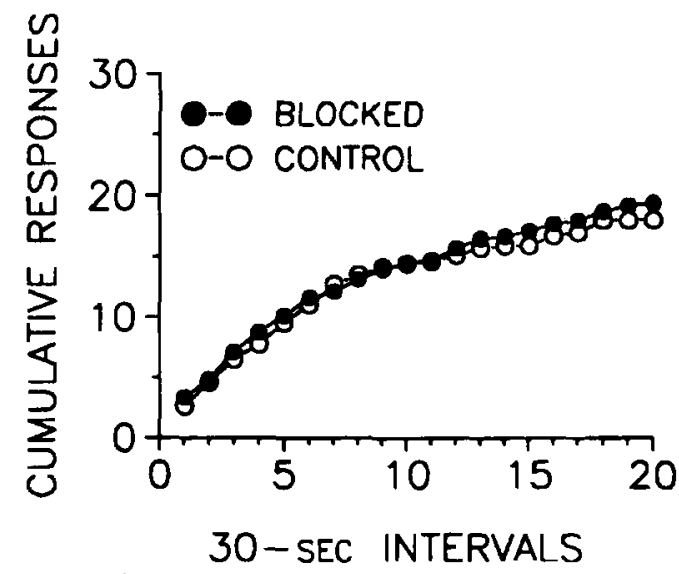

Figure 10. Performance in a nonreinforced choice test with the prospectively blocked and control odors of Experiment 6 .

(the second) yielded a treatment effect, which took the form of a very small, although statistically significant, interaction, and the results of subsequent efforts to find a set of experimental conditions under which the effect might be reproduced and magnified were entirely unsuccessful. If there is, in fact, some departure from strict independence in the conditioning of honeybees with color-odor compounds, it must be a rather modest one, as suggested to begin with by the fact that the data of a wide range of fairly complex color-odor experiments have been modeled successfully in disregard of that possibility (Couvillon \& Bitterman, 1991).

Although the results of experiments with color-odor compounds give little reason for dissatisfaction with the independence assumption, there are some earlier results for color-position compounds that are not easily reconciled with the assumption. In a color-position experiment like the color-odor experiment by Couvillon and Bitterman (1989) already described, Couvillon, Klosterhalfen, and Bitterman (1983) found reciprocal overshadowing in a confounded problem (e.g., green-left positive and blue-right negative) that was no easier than the colorrelevant problem (with position irrelevant) or the position-relevant problem (with color irrelevant). Less was learned about color in the confounded training than in the color-relevant training and less about position in the confounded training than in the position-relevant training, although experience with the relevant stimuli was in each case the same. Why the confounded problem should have been no easier than the other two-that is, why there was no evidence of summation as there was with colors and odors-and why the confounded groups should nevertheless have performed more poorly in the color and position tests are questions that remain to be investigated.

A still earlier experiment (Klosterhalfen, Fischer, \& Bitterman, 1978) showed positive dimensional transfer-another vertebrate phenomenon that the simple honeybee model fails to predict-in color-position but not in colorodor problems. Foragers trained first in a choice problem with color relevant or with position relevant and then in a second such problem with new colors and positions performed better in the second problem if the relevant dimension was the same in the two problems than if it was different; but after training in a color-odor problem with color or odor relevant, performance in a second problem. with new colors and odors was the same whether the relevant dimension was the same or different. If blocking could be demonstrated in color-position experiments analogous to those reported here, our negative results for color-odor compounds could not readily be discounted (as it might be tempting to do) on the assumption of unspecified weaknesses in technique or design.

Even now, however, it seems useful to consider why odor and position may not function in the same way when differentially reinforced in compound with color. Recent work with honeybees suggests that what has loosely been referred to in these experiments (and traditionally also in vertebrate choice experiments) as the "position" of a target may under some circumstances be given in relation to the visual surround and under others to position in the animal's visual field as determined by the animal's orientation (Huber, Couvillon, \& Bitterman, 1994; Wehner, 1981). In neither case is position an integral property of a target, as are its color or odor, and it is possible therefore that position competes in a special way with color and odor for control of performance. A distinctive manner of choice in position problems was, in fact, reported in both of the earlier papers (Couvillon, et al., 1983; Klosterhalfen, et al., 1978).

Another possibility, suggested by the fact that color and position are given visually, is that intramodal independence is less likely than intermodal independence. Much the same proposal has been made by Kehoe, Horne, Horne, and Macrae (1994) on the basis of results for tone-noise compounds versus tone-light and noise-light compounds obtained in conditioning experiments with rabbits. Whether the discrepant honeybee results for color-position versus color-odor compounds are better understood in terms of the unimodality of color-position compounds or in terms of their disjunctive character might be determined in experiments of the same kind with odor-position compounds. Support for the former interpretation is provided by evidence of blocking obtained recently in experiments on proboscis-extension conditioning in harnessed foragers by Smith and Cobey (1994), who used binary odor mixtures that were treated as odor-odor compounds.

\section{REFERENCES}

Couvillon, P. A., \& Bitterman, M. E. (1980). Some phenomena of associative learning in honeybees. Journal of Comparative \& Physiological Psychology, 94, 878-885.

Couvillon, P. A., \& Bitterman, M. E. (1982). Compound conditioning in honeybees. Journal of Comparative \& Physiological Psychology, 96, 192-199.

Couvillon, P. A., \& Bitterman, M. E. (1984). The overlearningextinction effect and successive negative contrast in honeybees (Apis mellifera). Journal of Comparative Psychology, 98, 100-109.

Couvillon, P. A., \& Bitterman, M. E. (1987). Discrimination of colorodor compounds by honeybees: Tests of a continuity model. Animal Learning \& Behavior, 15, 218-227. 
Couvillon, P. A., \& Bitterman, M. E. (1988). Compound-component and conditional discrimination of colors and odors by honeybees. $A n-$ imal Learning \& Behavior, 16, 67-74.

Couvillon, P. A., \& Bitterman, M. E. (1989). Reciprocal overshadowing in the discrimination of color-odor compounds by honeybees: Further tests of a continuity model. Animal Learning \& Behavior, 17, 213-222.

Couvillon, P. A., \& Bitterman, M. E. (1991). How honeybees make choices. In J. L. Goodman \& R. C. Fischer (Eds.), The behaviour and physiology of bees (pp. 116-130). Wallingford U.K.: CAB International.

Couvillon, P. A., Klosterhalfen, S., \& Bitterman, M. E. (1983). Analysis of overshadowing in honeybees. Journal of Comparative Psychology, 97, 154-166.

Durlach, P. J., \& Rescorla, R. A. (1980). Potentiation rather than overshadowing in flavor-aversion learning. Journal of Experimental Psychology: Animal Behavior Processes, 6, 175-187.

Fischer, M. E., Couvillon, P. A., \& Bitterman, M. E. (1993). Choice in honeybees as a function of the probability of reward. Animal Learning \& Behavior, 21, 187-195.

Frisch, K. vON. (1914). Der Farbensinn und Formensinn der Biene Zoologische Jahrbücher (Zoologie und Physiologie der Tiere), 35 1-182.

Huber, B., Couvillon, P. A., \& Bitterman, M. E. (1994). Place and position learning in honeybees (Apis mellifera). Journal of Comparative Psychology, 108, 213-219.

Hut.L, C. L. (1943). Principles of behavior. New York: Appleton-Century.

KAMIN, L. J. (1968). "Attention-like" processes in classical conditioning. In M. R. Jones (Ed.), Miami symposium on the prediction of behavior: Aversive stimulation (pp. 9-31). Miami: University of Miami Press.

Kehoe, E. J., Horne, A. J., Horne, P. S., \& Macrae, M. (1994). Summation and configuration between and within sensory modalities in classical conditioning of the rabbit. Animal Learning \& Behavior, 22, 19-26.

Klosterhalfen, S., Fischer, W., \& Bitterman, M. E. (1978, September 29). Modification of attention in honeybees. Science, 210 1241-1243.

LASHLEY, K. S. (1938). Conditional reactions in the rat. Joumal of PSVchology, 6, 311-324.

Loo, S. K., \& Bitterman, M. E. (1992). Learning in honeybees (Apis mellifera) as a function of sucrose concentration. Journal of Comparative Psychologi, 106, 29-36.

Pavlov, I. P. (1927). Conditioned reflexes: An investigation of the physiological activity of the cerebral cortex (G. V. Anrep, Ed. and Trans.). London: Oxford University Press.
RESCORLA, R. A. (1971). Variations in the effectiveness of reinforcement and nonreinforcement following prior inhibitory conditioning. Learning \& Motivation, 2, 113-123.

RESCORLA, R.A. (1972). "Configural" conditioning in discrete-trial bar pressing. Journal of Comparative \& Physiological Psychology, 79, 307-317.

ResCorla, R. A. (1981). Within-signal learning in autoshaping. Animal Learning \& Behavior, 9, 245-252.

Rescorla, R. A., \& Colwill, R. M. (1983). Within-compound associations in unblocking. Journal of Experimental Psychology: Animal Behavior Processes, 9, 390-400.

Rescorla, R. A., \& Cunningham, C. L. (1978). Within-compound flavor associations. Journal of Experimental Psychology: Animal Behavior Processes, 4, 267-275.

Rescorla, R. A., \& Wagner, A. R. (1972). A theory of classical conditioning: Variation in the effectiveness of reinforcement and nonreinforcement. In A. H. Black \& W. F. Prokasy (Eds.), Classical conditioning II: Current research and theory (pp. 64-99). New York: Appleton-Century-Crofts.

Shinoda, A., \& BitTerman, M. E. (1987). Analysis of the overlearningextinction effect in honeybees. Animal Learning \& Behavior, 15, 93 96.

Smith, B. H., \& CoвEy, S. (1994). The olfactory memory of the honeybee. Apis mellifera. Journal of Experimental Biology, 195, 91-108.

Sutherland, N. S., \& Mackintosh, N. J. (1971). Mechanisms of animal discrimination learning. New York: Academic Press.

WAGNER, A. R. (1969). Incidental stimuli and discrimination learning. In R. M. Gilbert \& N. S. Sutherland (Eds.), Animal discrimination learning (pp. 83-111). New York: Academic Press.

WAGNER, A. R. (1971). Elementary associations. In H. H. Kendler \& J. T. Spence (Eds.), Essays in neobehaviorism (pp. 187-213). New York: Appleton-Century-Crofts.

WEHNER, R. (1981). Spatial vision in arthropods. In H. Autrum (Ed.), Comparative physiology and evolution of vision in invertebrates. $C$ : Invertebrate visual centers and behavior II (pp. 287-616). Berlin: Springer-Verlag.

WhITLOW, J. W., JR., \& WAGNER, A. R. (1972). Negative patterning in classical conditioning: Summation of response tendencies to isolable and configural components. Psychonomic Science, 27, 299-301.

(Manuscript received August 2, 1994; revision accepted for publication November 24, 1994.) 